\title{
Differential Anthocyanin Concentrations and Expression of Anthocyanin Biosynthesis Genes in Strawberry 'Sachinoka' during Fruit Ripening under High-temperature Stress
}

\author{
Keisuke OKuTsu, Kohei Matsushita and Takashi IKedA \\ School of Agriculture, Meiji University, Kawasaki, Kanagawa 214-8571, Japan
}

(Received January 31, 2017; Accepted September 5, 2017)

\begin{abstract}
We investigated anthocyanin concentration and the expression of anthocyanin biosynthesis related genes in strawberry 'Sachinoka' (Fragaria $\times$ ananassa Duch.) grown at control regime $\left(20 / 15^{\circ} \mathrm{C}, 14 / 10 \mathrm{~h}\right.$, day/night) or high temperature regime $\left(30 / 15^{\circ} \mathrm{C}, 14 / 10 \mathrm{~h}\right)$. In Experiment 1 , fruits were harvested at different color stages. In the control treatment, the anthocyanin concentration increased as fruit ripened, and was highest at the fully ripe stage. In the high-temperature treatment, however, it was significantly lower at the fully ripe stage. These results were relevant with the peak value of expression of anthocyanin biosynthesis genes which was inhibited in fruits grow at high temperature regime. In Experiment 2, we applied high temperature at different fruit color stages to test the effect of the timing of high temperature stress on coloring. When exposed fruit to high temperature after half ripe stage, the anthocyanin concentration and gene expression in fully ripe fruit were not significantly different from the control. But in fruit exposed before half ripe stage, the anthocyanin concentration and gene expression significantly reduced. These results indicate that the level of gene expression of anthocyanin biosynthesis is affected by the timing of exposure fruit to high temperature stress.
\end{abstract}

Keywords : fruit coloring stage, gene expression, timing of stress

\section{INTRODUCTION}

Fruit color is an important factor in fruit quality. Anthocyanins are common pigments that accumulate in many fruits, including grape, apple, and strawberry. The regulation of their biosynthesis has been studied widely and frequently (review in Jaakola, 2013). In particular, UFGT (UDP-glucose: flavonoid 3-O-glucosyltransferase) plays an important role in anthocyanin biosynthesis in grape (Boss et al., 1996; Kobayashi, 2009), and FaMYB10 regulates the expression of FaUFGT in strawberry (Medina-Puche et al., 2014; Kadomura-Ishikawa et al., 2015a).

It is well known that anthocyanin accumulation in fruits is affected by temperature, and is decreased when fruits are grown at high temperature (Saure, 1990; Ikeda et al., 2011). Recently, we demonstrated that the anthocyanin concentration and the expression of genes related to anthocyanin biosynthesis decreased in strawberry 'Sachinoka' fruits grown at high temperature (Matsushita et al., 2016). In grape, high temperature inhibits anthocyanin accumulation by reducing the activity of UFGT (Mori et al., 2004). In apple, rapidly reduces the expression of the R2R3MYB transcription factor (TF) gene (MYB10), which regulates the coordination of red skin coloration (Lin-Wang et al., 2011).

Although high temperature stress could occur at any time during fruit coloring, published studies of the effect on anthocyanin biosynthesis applied high temperature throughout the coloring period (Mori et al., 2004; Matsushita et al., 2016), but not at different ripening stages. There are two studies examined the effect of temperature on coloring in grapes and rose: Yamane et al. (2006) investigated the temperature-sensitive stages for coloration and the mechanisms that underlie the effect of temperature on anthocyanin accumulation using 2-week treatments at 20 and $30^{\circ} \mathrm{C}$. Then, they elucidated that stage 3 (from one to three weeks after the onset of coloring) is the most sensitive for anthocyanin accumulation in the berry skins of 'Aki Queen'. Dela et al. (2003) described the effect of transient high temperature on the concentration and composition of anthocyanins in 'Jaguar' rose flowers, and showed that although a $1-\mathrm{d}$ high temperature treatment $\left(39 / 18^{\circ} \mathrm{C}\right.$ day/ night) had no effect on pigmentation, a 3-d treatment had a substantial effect on pigmentation due to decreasing anthocyanin accumulation. However, we cannot find any report of the effect of applying high temperature at different coloring stages or of the relationship between anthocyanin concentration and the expression of genes related to anthocyanin biosynthesis in strawberry.

Therefore, we designed two experiments to explore this. Experiment 1 investigated the influence of high air temperature $\left(30 / 15^{\circ} \mathrm{C}\right)$ on anthocyanin biosynthesis at different coloring stages. Experiment 2 examined the effect of high air temperature in fruits exposed to the high temperature at different coloring stages and harvested when fully ripe.

Corresponding author: Takashi Ikeda, fax: $+81-44-934-7902$, e-mail : agrisys@meiji.ac.jp 


\section{MATERIALS AND METHODS}

\section{Plant materials}

Fragaria $\times$ ananassa Duch 'Sachinoka' were planted in $10.5-\mathrm{cm}$ pots in commercial horticultural soil (Housakumonogatari, Takii \& Asahi, Tokyo, Japan). The plants were grown in a greenhouse, watered every day, and supplied with fertilizer according to standard practice. The flowers were pollinated by honeybees. After pollination, we trimmed the plants to a single flower and moved them into growth chambers (HNM-S, Koito Industries, Tokyo, Japan) set to either the control regime $\left(20 / 15^{\circ} \mathrm{C}\right.$ day/night) or the high-temperature regime $\left(30 / 15^{\circ} \mathrm{C}\right)$, with $80 \%$ relative humidity, 14/10-h (day/night) photoperiod, and a photosynthetic photon flux density of $190 \mu \mathrm{mol} \mathrm{m} \mathrm{m}^{-2} \mathrm{~s}^{-1}$. In Experiment 1, fruits were harvested at the green (G: almost $16 \mathrm{~d}$ after pollination (DAP)), slightly ripe (SR: almost 20 DAP), half ripe (HR: almost 23 DAP), or fully ripe (FR: almost 29 DAP) coloring stage (Fig. 1) in control regime. In Experiment 2, plants were grown in the control regime until harvest $(\mathrm{CT})$, in the high-temperature regime until harvest (HT), or in the control regime until G, SR, HR, or before FR (BFR: almost 26 DAP; Fig. 1) and then moved into the high temperature regime and grown until harvest (FR). The harvested fruits were frozen in liquid nitrogen and stored at $-80^{\circ} \mathrm{C}$.
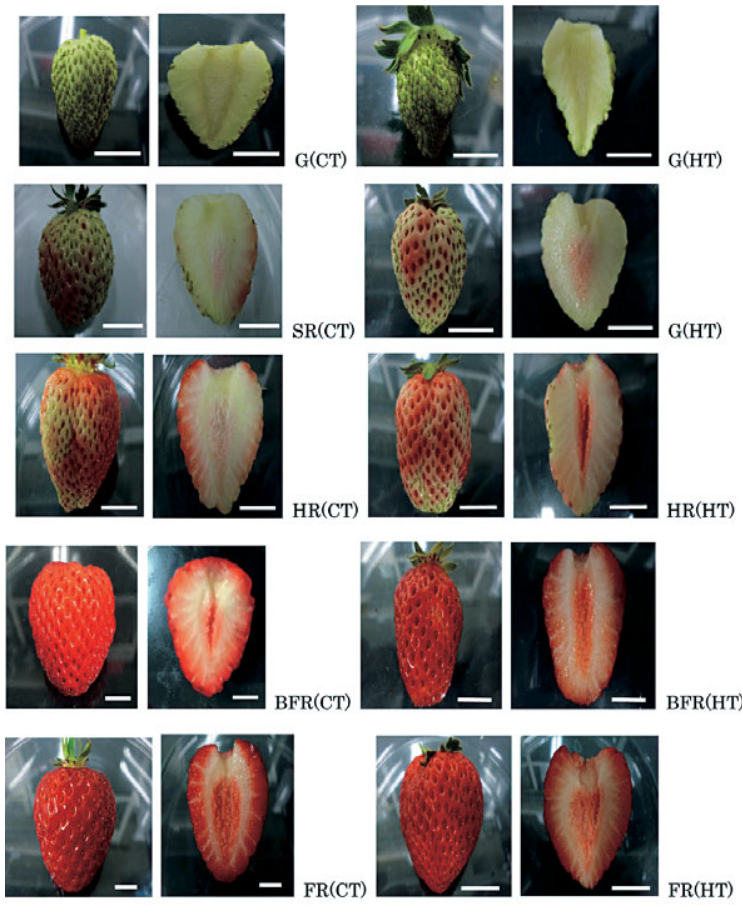

Fruit surface longitudinal section
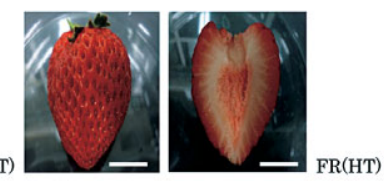

Fruit surface longitudinal section

Fig. 1 Pigmentation stages of strawberry. Green (G); Slightly ripe (SR); half ripe (HR); Before fully ripe (BFR); Fully ripe (FR). CT means control regime and HT means high temperature regime. In the following figures, the pigmentation stages of fruit are shown by the same capitals. White bars in pictures are approximately $10 \mathrm{~mm}$.
Anthocyanin measurement

We measured anthocyanin concentration based on the method of Pombo et al. (2011). Samples were powdered in liquid nitrogen, and then $0.1 \mathrm{~g}$ was vortexed in $1 \mathrm{~mL}$ of HCl-methanol. The samples were chilled on ice for $>2$ min, and then centrifuged at $12,000 \times \mathrm{g}$ for $15 \mathrm{~min}$ at room temperature. The absorbance of the supernatant was measured with a spectrophotometer (NanoDrop 2000, Thermo Fisher Scientific Inc., Waltham, MA, USA) and converted to molar concentration by the method of Matsushita et al. (2016). Three separate measurements of three to six fruits were taken.

RNA extraction and quantitative PCR

RNA was extracted as described by Reid et al. (2006). The RNA concentration was measured with NanoDrop 2000, cDNA was synthesized from the isolated RNA using a PrimeScript RT Reagent Kit with gDNA Eraser (Perfect Real Time; Takara Bio, Tokyo, Japan). We investigated the anthocyanin biosynthesis genes CHI (chalcone isomerase; HM026182), F3H (flavanone 3-hydroxylase; HM026183), CHS (chalcone synthase; AY997297), DFR (dihydroflavonol 4-reductase; AF029685), ANS (anthocyanidin synthase; AY695817), and UFGT (AY575056), and the MYB TF genes FaMYB1 (AF401220), FaMYB5 (JQ989280), and FaMYB10 (EU155162) in Experiment 1; and all but $\mathrm{CHI}$ and $\mathrm{F3H}$ in Experiment 2. Table 1 shows sequences of the primers in this study. The primers were based on sequences provided in the GenBank database, and designed using Primer3 primer design software. The others were quoted from Kadomura-Ishikawa et al. (2015a in supplementary material 3). Quantitative PCR (qPCR) was performed on a Real-Time PCR System (7300 Applied Biosystems, Foster City, CA, USA), with three technical replicates per sample. Gene expression was expressed relative to actin (AB116565) (Almeida et al., 2007; Schmittgen and Livak, 2008; Matsushita et al., 2016).

Table 1 Sequences of the primers used in this study.

\begin{tabular}{|c|c|c|}
\hline \multicolumn{2}{|c|}{ Name } & \multirow{2}{*}{$\frac{\text { Sequences }\left(5^{\prime} \text { to } 3 \text { ') }\right.}{\text { GCTGTCAAGGCCATTAAGGA }}$} \\
\hline & forward & \\
\hline CHS & reverse & GAGCAAACAACGAGAACACG \\
\hline \multirow{2}{*}{$\mathrm{CHI}$} & forward & TTTTCAATGGCTTTCGCTTCTG \\
\hline & reverse & GTGACAATGATACTACCGCTGACG \\
\hline \multirow{2}{*}{ F3H } & forward & GTGCGCCACCGTGACTACTC \\
\hline & reverse & ATGCCTTTGTCAATGCCTCC \\
\hline \multirow{2}{*}{$D F R$} & forward & GTAGAGGTGATTCAGAGTCTCC \\
\hline & reverse & CTTCCACTAGCTGAAGGAAGTTC \\
\hline \multirow{2}{*}{ ANS } & forward & GACTTGTCCATTTGGCCTC \\
\hline & reverse & СССССТCAGTTCCTTAGCATACTC \\
\hline \multirow{2}{*}{$U F G T$} & forward & CAAGCAATCCAACAGCTCAA \\
\hline & reverse & TGTCAAGCCACGATAAGCAG \\
\hline \multirow{2}{*}{ FaMYBI } & forward & AGACTGCCTGGAAGGACAGA \\
\hline & reverse & TTCTCATGGGGCTTATTTGG \\
\hline \multirow{2}{*}{ FaMYB5 } & forward & AGCAAGCCAGCACCAGTTG \\
\hline & reverse & CCATTAGGCTGTTGCATTACTTGTA \\
\hline \multirow{2}{*}{ FaMYB10 } & forward & TTTAATTGCCGGAAGATTGC \\
\hline & reverse & GGTTCGTGGTCGAGGTCTTA \\
\hline \multirow{2}{*}{ Actin } & forward & TGTGGCCATTCAAGCTGTTC \\
\hline & reverse & TCACCAGAGTCGAGCACAAT \\
\hline
\end{tabular}




\section{RESULTS}

\section{Experiment 1}

At FR, the anthocyanin concentration of fruits grown under the high-temperature regime was only $40 \%$ of that under the control regime (Fig. 2). At SR and HR, the difference was not significant, and at $\mathrm{G}$, anthocyanin was not detected. The expression of all genes related to anthocyanin biosynthesis increased with fruit color development from G to HR (Fig. 3), and then decreased at FR. At HR, the expression of all genes was significantly lower in the high-temperature regime, but by FR, only lateregulated biosynthesis genes (LBGs: DFR, ANS, UFGT) were significantly lower, in particular, the expression of $U F G T$ was halved in the high-temperature regime (Fig. 3). The expression of FaMYB1 and FaMYB5 did not differ significantly at any color stage, but that of FaMYB10 was significantly reduced in the high-temperature regime at both HR (45\% of control) and FR (36\% of control), similar to the UFGT result (Figs. 3, 4).

\section{Experiment 2}

Fruit length had no difference in all regimes (Table 2). Fruit weight in HT had almost half $(52 \%)$ of that in CT (Table 2).

The anthocyanin concentration of fruits transferred immediately (HT) or at G and SR was markedly less than that of fruits held under CT. That of fruits transferred to the high-temperature regime at different coloration stages no effect at HR and BFR (Fig. 5). In particular, that at HT decreased until $45 \%$ of CT. The anthocyanin biosynthesis genes DFR and UFGT and the FaMYB10 TF showed the same pattern (Figs. 6,7): the level of UFGT expression was $45 \%$ of CT at HT (Fig. 6), and that of FaMYB10 expression was only $21 \%$ of CT at HT, G, and SR (Fig. 7).

\section{DISCUSSION}

The effects of external factors on anthocyanin biosyn-

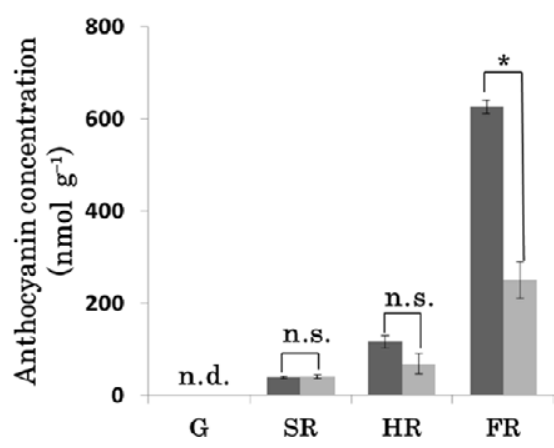

Fig. 2 Anthocyanin concentration in Experiment 1. Dark gray bars show control regime $\left(20^{\circ} \mathrm{C}\right)$; light gray bars show high-temperature regime $\left(30^{\circ} \mathrm{C}\right)$. Data are means $\pm \mathrm{SE}$ (standard error) $(n=3)$. *Significantly different between control and high temperature at $P<0.05$, and n.s. indicates not significant at $P>0.05$ by Student's $t$ test. Anthocyanin in $\mathrm{G}$ was not detected (n.d.). G, SR, HR, FR means harvested time of strawberry fruits in both regimes. thesis and regulation are becoming clearer (Jeong et al., 2004; Castellarin et al., 2007; Azuma et al., 2012). Jeong et al. (2004) reported that the accumulation of anthocyanins in grape fruit was enhanced by abscisic acid treatment and suppressed by naphthaleneacetic acid and shading. Kadomura-Ishikawa et al. (2015a) suggested that light and abscisic acid independently regulate anthocyanin biosynthesis via activation of FaMYB10 expression in strawberry fruits. Azuma et al. (2012) elucidated that the expression of other MYB-related genes in grape and of many flavonoid biosynthesis pathway genes was regulated independently by temperature and light. Castellarin et al. (2007) showed that water stress to grape fruits at the early developmental stage promoted maturity and anthocyanin biosynthesis. We recently investigated the effect of water stress on strawberry fruits and confirmed that it promoted the accumulation of anthocyanins and maturation, but that it inhibited fruit development (Ikeda et al., 2016). Here, we investigated the effect of the timing of high temperature.

The anthocyanin concentration in fruits increased from none at the $\mathrm{G}$ stage to maximum at the FR stage (Fig. 2). This timing is relevant to the increased anthocyanin biosynthesis-related genes which peaked at the HR stage and then

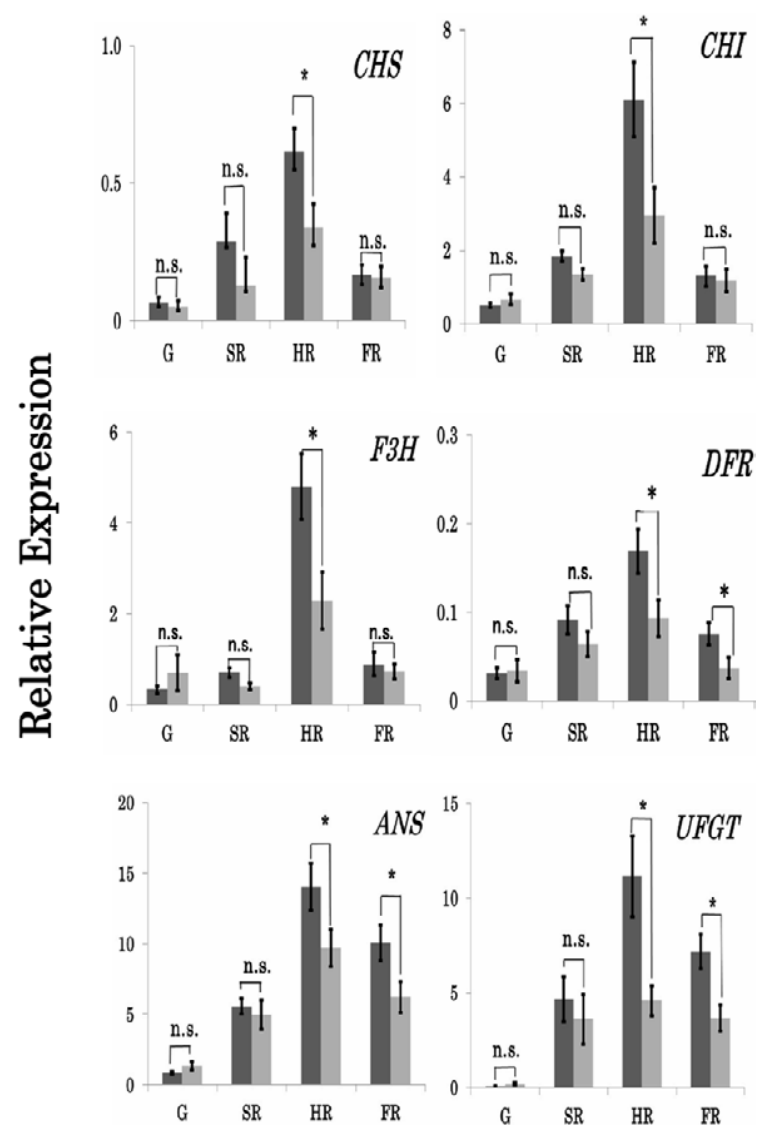

Fig. 3 Relative expression of anthocyanin synthesis genes in Experiment 1. Dark gray bar show control regime $\left(20^{\circ} \mathrm{C}\right)$; light gray bar show high-temperature regime $\left(30^{\circ} \mathrm{C}\right)$. Values are relative expression levels normalized to $\beta$-actin. Data are means $\pm \operatorname{SE~}(n=3)$. *Significantly different between control and high temperature at $P<0.05$, and n.s. indicates not significant at $P>0.05$ by Student's $t$ test. G, SR, HR, FR means harvested time of strawberry fruits in both regimes. 
K. OKUTSU ET AL.
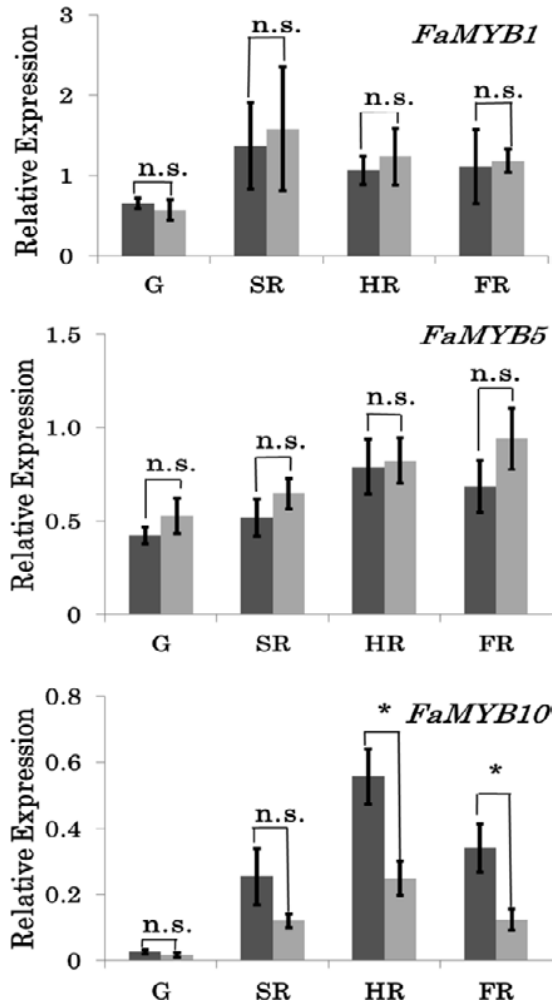

Fig. 4 Relative expression of $M Y B$ genes in Experiment 1. Dark gray bar show control regime $\left(20^{\circ} \mathrm{C}\right)$; light gray bar show high-temperature regime $\left(30^{\circ} \mathrm{C}\right)$. Values are relative expression levels normalized to $\beta$-actin. Data are means $\pm \operatorname{SE}(n=3)$. *Significantly different between control and high temperature at $P<0.05$, and n.s. indicates not significant at $P>0.05$ by Student's $t$ test. G, SR, HR, FR means harvested time of strawberry fruits in both regimes.

fell at the FR stage (Fig. 3). This pattern implies that anthocyanin in fruits was rapidly synthesized between HR and FR stage, because the expression of all anthocyanin biosynthesis related genes except FaMYBI and FaMYB5 had peaked at HR (Figs. 3, 4). The anthocyanin concentration was significantly difference between high temperature regime and control regime (Fig. 2), as a consequence of the expression level at high temperature regime was significantly low (Fig. 3). In FR stage, the expression level for LBGs and FaMYB10 still showed significant difference (Figs. 3, 4).

In Experiment 2, we considered the effect of the timing of high temperature stress on the coloring of strawberry fruits. Yamane et al. (2006) investigated the effect of high $\left(30^{\circ} \mathrm{C}\right)$ and low $\left(20^{\circ} \mathrm{C}\right)$ temperatures at different stages of development and ripening on the accumulation of anthocyanins in berry skins of a red table grape, "Aki Queen'. They described that the high and low temperatures

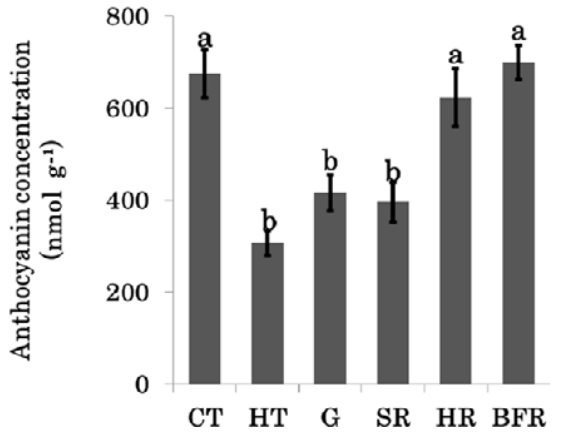

Fig. 5 Anthocyanin concentrations in Experiment 2. Data are means $\pm \mathrm{SE}(n=5)$. Bars marked with the same letter are not significantly different at $P<0.05$ by TukeyKramer test. G, SR, HR, BFR means transferred time of strawberry fruit from control regime to high temperature regime. FR means cultured in control regime until harvested time.

during ripening, especially in stage 3 (from one to three weeks after the onset of coloring) affects the expression of $V v M Y B A 1$; the product of $V v M Y B A 1$ then controls the expression of the anthocyanin biosynthetic enzyme genes. Dela et al. (2003) showed that the temperature sensitivity of the anthocyanin concentration and composition of 'Jaguar' rose flowers to transient high temperatures depended on the developmental stage. Nevertheless, the effects on strawberry fruits have been little considered, probably because strawberries mature in only 20 to $30 \mathrm{~d}$ after pollination. We investigated the effect of imposing high temperature on strawberry fruits at different coloring stages on the fully ripe color. Fruits moved into the high-temperature regime before the HR stage had almost the same anthocyanin concentration as those grown entirely under the hightemperature regime, whereas fruits moved at or after the HR stage had almost the same concentration as those grown entirely under the control regime (Fig. 5). These results show that the timing of exposure to high temperature strongly influenced the final anthocyanin concentration.

In Experiment 1, the anthocyanin biosynthesis related genes were expressed most strongly at the HR stage (Figs. 3 , 4). In experiment 2, DFR, UFGT, and FaMYB10 were expressed more strongly in fruits transferred to the high temperature regime at the HR stage than in those transferred at the SR stage (Figs. 6, 7). Thus, the level of expression of the LBGs except ANS had peaked at HR stage, then, even the high temperature stress was applied after HR stage, the level was similar to that of control regime (Figs. $6,7)$.

The expression of CHS showed no significant difference at the FR stage in either experiment (Figs. 3, 6). The expression of $\mathrm{CHI}$ and $\mathrm{F} 3 \mathrm{H}$ showed no significant difference either (Fig. 3). In Experiment 2, the anthocyanin bio-

Table 2 Fruit weight (g FW) and fruit length (mm) for the fruits which were used for Experiment 2.

\begin{tabular}{crrrrrc}
\hline & \multicolumn{1}{c}{ CT } & \multicolumn{1}{c}{ HT } & \multicolumn{1}{c}{ G } & \multicolumn{1}{c}{ SR } & HR & BFR \\
\hline Fruit weight (g FW) & $16.4 \pm 1.0$ & $8.5 \pm 0.8$ & $9.1 \pm 0.7$ & $10.6 \pm 0.7$ & $13.4 \pm 0.5$ & $14.7 \pm 0.8$ \\
Fruit length (mm) & $34.8 \pm 1.2$ & $29.4 \pm 1.2$ & $31.0 \pm 0.6$ & $30.5 \pm 1.0$ & $32.4 \pm 0.7$ & $33.7 \pm 0.5$ \\
\hline
\end{tabular}

Datas are mean \pm standard error $\left(n=5^{-7}\right)$. CT; control regime until harvest, HT; the high-temperature regime until harvest, G; green stage, SR; slightly ripe stage, HR; half ripe stage, BFR; before fully ripening. 

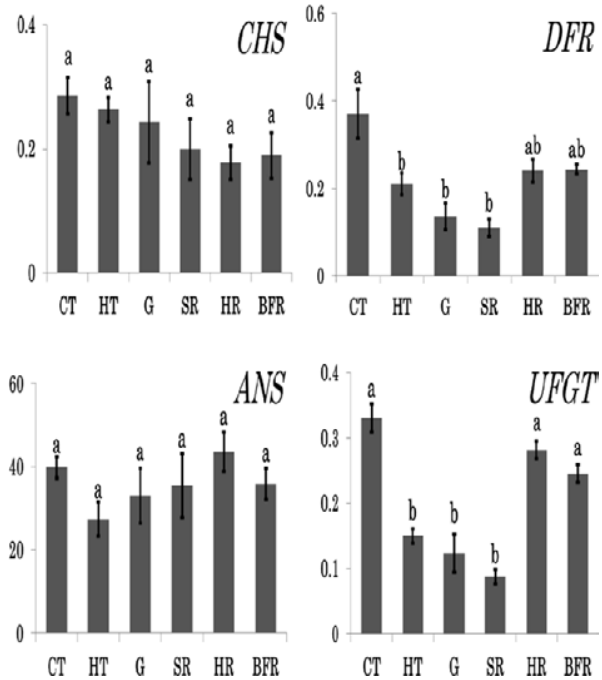

Fig. 6 Relative expression of anthocyanin synthesis genes in Experiment 2. Values are relative expression levels normalized to $\beta$-actin. Data are means $\pm \operatorname{SE}(n=5)$. Bars marked with the same letter are not significantly different at $P<0.05$ by Tukey-Kramer test. G, SR, HR, BFR means transferred time of strawberry fruit from control regime to high temperature regime. FR means cultured in control regime until harvested time.
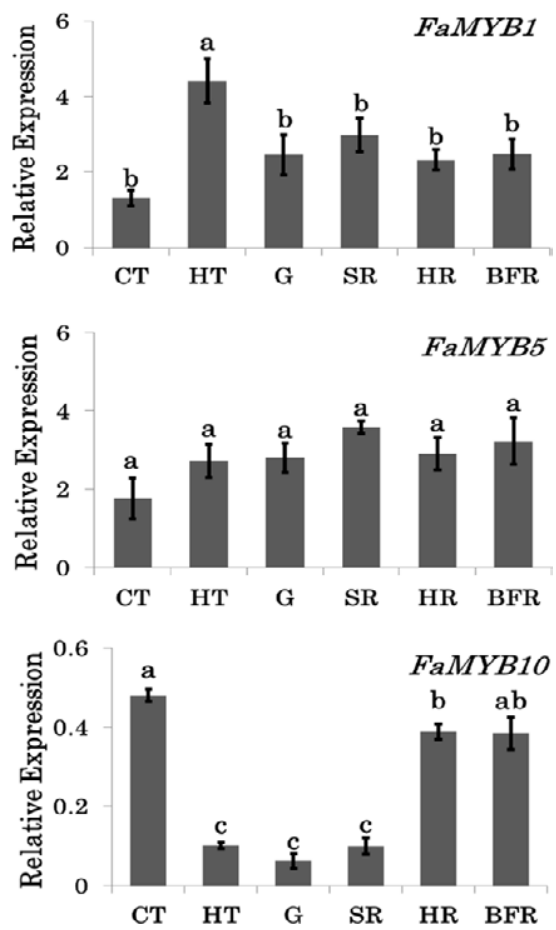

Fig. 7 Relative expression of $M Y B$ genes in Experiment 2. Values are relative expression levels normalized to $\beta$ actin. Data are means $\pm \mathrm{SE}(n=5)$. Bars marked with the same letter are not significantly different at $P<0.05$ by Tukey-Kramer test. G, SR, HR, BFR means transferred time of strawberry fruit from control regime to high temperature regime. FR means cultured in control regime until harvested time.

synthesis genes DFR and UFGT showed close agreement with anthocyanin concentration, and were significantly decreased under the high-temperature regime in HT, G, and
SR (Fig. 6). This effect might be induced by MYB TFs, as the expression of FaMYB10 was similar to that of DFR and $U F G T$, but not ANS (Figs. 6, 7). Medina-Puche et al. (2014) stated that FaMYB10 is not involved in the expression of ANS. Kadomura-Ishikawa et al. (2015a) stated that FaMYB10 is deeply involved in UFGT expression. Our results agree with those studies.

The regulation of anthocyanin biosynthesis by MYB TFs has been studied using MYB cDNAs (Aharoni et al., 2001; Schaart et al., 2013; Kadomura-Ishikawa et al., 2015a). Transgenic tobacco transformed with FaMYBI isolated from strawberry fruits showed a severe reduction in pigmentation and in the expression of late flavonoid biosynthesis genes, and FaMYB1 overexpression adversely affected the activities of the late flavonoid biosynthesis enzymes (Aharoni et al., 2001). In contrast, FaMYB10 overexpression may have increased anthocyanin content by upregulating anthocyanin biosynthesis genes (KadomuraIshikawa et al., 2015a). Whereas FaMYB1 is dominant in unripe fruit, FaMYB10 becomes dominant with ripening, accelerating anthocyanin accumulation (KadomuraIshikawa et al., 2015b). We suggested that the expression level of FaMYB1 was promoted by exposed high temperature at early period of maturity in this study. The function of FaMYB10 is obviously related to the regulation of anthocyanin biosynthesis, and our results support the hypothesis that decreased expression of FaMYB1O is related closely to the decreased anthocyanin concentration.

In conclusion, the results of Experiment 1 suggest that high temperature inhibited the expression of anthocyanin biosynthesis genes at and after the HR stage, and the results of Experiment 2 shows that anthocyanin concentration and the expression of anthocyanin biosynthesis genes significantly decreased in fruits exposed to high temperature stress immediately (HT) and at the G and SR stages. Both results suggest that $U F G T$ and FaMYB1O play important roles in anthocyanin biosynthesis, regardless of the timing of harvest or of high temperature stress.

\section{REFERENCES}

Aharoni, A., De Vos, R. C. H., Wein, M., Sun, Z., Greco, R., Kroon, A., Mol, J. N. M., O'Connell, A. P. 2001. The strawberry FaMYB1 transcription factor suppresses anthocyanin and flavonol accumulation in transgenic tobacco. Plant J. 28: 319-332.

Almeida, J. R. M., D’Amico, E., Preuss, A., Carbone, F., De Vos, R. C. H., Deiml, B., Mourgues, F., Perrotta, G., Fischer, T. C., Bovy, A. G., Martens, S., Rosati, C. 2007. Characterization of major enzymes and genes involved in flavonoid and proanthocyanidin biosynthesis during fruit development in strawberry (Fragaria $\times$ ananassa). Arch. Biochem. Biophys. 465: 61-71.

Azuma, A., Yakushiji, H., Koshita, Y., Kobayashi, S. 2012. Flavonoid biosynthesis-related genes in grape skin are differentially regulated by temperature and light conditions. Planta 236: $1067-1080$.

Boss, P. K., Davies, C., Robinson, S. P. 1996. Expression of anthocyanin biosynthesis pathway genes in red and white grapes. Plant Mol. Biol. 32: 565-569.

Castellarin, S. D., Matthews, M. A., Di Gaspero, G., Gambetta, G. 


\section{K. OKUTSU ET AL.}

A. 2007. Water deficits accelerate ripening and induce changes in gene expression regulating flavonoid biosynthesis in grape berries. Planta 227: 101-112.

Dela, G., Or, E., Ovadia, R., Nissim-Levi, A., Weiss, D., OrenShamir, M. 2003. Changes in anthocyanin concentration and composition in 'Jaguar' rose flowers due to transient high air temperature conditions. Plant Sci. 164: 333-340.

Ikeda, T., Suzuki, N., Nakayama, M., Kawakami, Y. 2011. The effects of high air temperature and water stress on fruit growth and anthocyanin concentration of pot-grown strawberry (Fragaria $\times$ ananassa Duch. cv. 'Sachinoka') plants. Environ. Control Biol. 49: 209-215.

Ikeda, T., Matsushita, K., Oyaizu, Y., Okutsu, K. 2016. Different expressions of anthocyanin biosynthetic genes for strawberry 'Sachinoka' fruits under water and high temperature stresses. 8th International Strawberry Symposium 2016, Quebec City, Canada. Book of Abstracts: 144.

Jaakola, L. 2013. New insights into the regulation of anthocyanin biosynthesis in fruits. Trends. Plant Sci. 18: 477483.

Jeong, S. T., Goto-Yamamoto, N., Kobayashi, S., Esaka, M. 2004. Effects of plant hormones and shading on the accumulation anthocyanins and the expression of anthocyanin biosynthetic genes in grape berry skins. Plant Sci. 167: 247-252.

Kadomura-Ishikawa, Y., Miyawaki, K., Takahashi, A., Masuda, T., Noji, S. 2015a. Light and abscisic acid independently regulated FaMYB10 in Fragaria $\times$ ananassa fruit. Planta 241: 953-965.

Kadomura-Ishikawa, Y., Miyawaki, K., Takahashi, A., Masuda, T., Noji, S. 2015b. RNAi-mediated silencing and overexpression of the FaMYBl gene and its effect on anthocyanin accumulation in strawberry fruit. Biol. Plantarum 59: 677685.

Kobayashi, S. 2009. Regulation of anthocyanin biosynthesis in grapes. J. Jpn. Soc. Hortic. Sci. 78: 387-393.

Lin-Wang, K., Micheletti, D., Palmer, J., Volz, R., Lozano, L., Espley, R., Hellens, R. P., Chagne, D., Rowan, D. D., Troggio, M., Iglesias, I., Allan, A. C. 2011. High air temperature reduces apple fruit color via modulation of the anthocyanin regulatory complex. Plant Cell Environ. 34: $1176-1190$.
Matsushita, K., Sakayori, T., Ikeda, T. 2016. The effect of high air temperature on anthocyanin concentration and the expressions of its biosynthetic genes in strawberry 'Sachinoka'. Environ. Control Biol. 54: 101-107.

Medina-Puche, L., Cumplido-Laso, G., Amil-Ruiz, F., Hoffmann, T., Ring, L., Rodríguez-Franco, A., Caballero, J. L., Schwab, W., Muñoz-Blanco, J., Blanco-Portales, R. 2014. MYB10 plays a major role in the regulation of flavonoid/ phenylpropanoid metabolism during ripening of Fragaria $\times$ ananassa fruits. J. Exp. Bot. 65: 401-417.

Mori, K., Sugaya, S., Gemma, H. 2004. Regulatory mechanism of anthocyanin biosynthesis in 'Kyoho' grape berries grown under different temperature conditions. Environ. Control Biol. 42: 21-30.

Mori, K., Goto-Yamamoto, N., Kitayama, M., Hashizume, K. 2007. Loss of anthocyanins in red-wine grape under high air temperature. J. Exp. Bot. 58: 1935-1945.

Pombo, M. A., Martínez, G. A., Civello, P. M. 2011. Cloning of FaPAL6 gene from strawberry fruit and characterization of its expression and enzymatic activity in two cultivars with different anthocyanin accumulation. Plant Sci. 181: 111-118.

Reid, K. E., Olsson, N., Schlosser, J., Peng, F., Lund, S. T. 2006. An optimized grapevine RNA isolation procedure and statistical determination of reference genes for real-time RT-PCR during berry development. BMC Plant Biol. 6: 27.

Saure, M. C. 1990. External control of anthocyanin formation in apple. Sci. Hortic. 42: 181-218.

Schaart, J. G., Dubos, C., De La Fuente, I. R., Van Houwelingen, A. M. M. L., de Vos, R. C. H., Jonker, H. H., Xu, W., Routaboul, J. M., Lepiniec, L., Bovy, A. G. 2013. Identification and characterization of MYB-bHLH-WD40 regulatory complexes controlling proanthocyanidin biosynthesis in strawberry (Fragaria $\times$ ananassa) fruits. New. Phytol. 197: 454-467.

Schmittgen, T. D., Livak, K. J. 2008. Analyzing real-time PCR data by the comparative CT method. Nature Protocols 3: 1101-1108.

Yamane, T., Jeong, S. T., Goto-Yamamoto, N., Koshita, Y., Kobayashi, S. 2006. Effect of temperature on anthocyanin biosynthesis in grape berry skins. Am. J. Enol. Vitic. 57: 5459. 